Thermal-wave balancing flow sensor with low-drift power feedback

This content has been downloaded from IOPscience. Please scroll down to see the full text.

2014 J. Micromech. Microeng. 24055016

(http://iopscience.iop.org/0960-1317/24/5/055016)

View the table of contents for this issue, or go to the journal homepage for more

Download details:

IP Address: 130.89.19.94

This content was downloaded on 14/01/2015 at $14: 14$

Please note that terms and conditions apply. 


\title{
Thermal-wave balancing flow sensor with low-drift power feedback
}

\author{
M Dijkstra, T S J Lammerink, O Pjetri, M J de Boer, J W Berenschot, \\ R J Wiegerink and M C Elwenspoek
}

Transducers Science and Technology Group, $\mathrm{MESA}^{+}$Institute for Nanotechnology, University of Twente, PO box 217, 7500 AE Enschede, The Netherlands

E-mail: m.a.dijkstra@ewi.utwente.nl

Received 15 January 2014, revised 27 February 2014

Accepted for publication 12 March 2014

Published 11 April 2014

\begin{abstract}
A control system using a low-drift power-feedback signal was implemented applying thermal waves, giving a sensor output independent of resistance drift and thermo-electric offset voltages on interface wires. Kelvin-contact sensing and power control is used on heater resistors, thereby inhibiting the influence of heater resistance drift. The thermal waves are detected with a sensing resistor using a lock-in amplifier and are mutually cancelled by a thermal-wave balancing controller. Offset due to thermal gradient across the chip and resistor drift are eliminated by the lock-in amplifier and power controller, and therefore do not influence the sensor output signal. A microchannel thermal-wave balancing flow sensor with integrated $\mathrm{Al}$ resistors has successfully been fabricated. The thermal flow sensor is capable of measuring water flow rates with $\mathrm{nl} \cdot \mathrm{min}^{-1}$ precision, up to about $500 \mathrm{nl} \cdot \mathrm{min}^{-1}$ full scale. Measurement results are in good agreement with a dynamic model of the flow sensor. Drift measurements show the sensor output signal to be compensated for resistance drift and thermal gradient across the chip.
\end{abstract}

Keywords: thermal waves, power feedback, flow sensor

(Some figures may appear in colour only in the online journal)

\section{Introduction}

Increased miniaturization of flow sensors has resulted in micro thermal flow sensors capable of measuring liquid flows down to a few $\mathrm{nl} \cdot \mathrm{min}^{-1}[1,2]$. These flow sensors require accurate measurement of very small flow induced temperature changes. Using a thermopile and power-feedback control [3] allows the reduction of sensor output dependency on drift in resistance values and drift in thermopile sensitivity. However, it is difficult to characterize and eliminate the influence of thermo-electric offset voltages, resistance drift, thermal gradients across the chip and other slowly varying influences on sensor output drift. For example, sensor output dependency on redistribution of spatial variations in resistivity of the heater resistors and irregularities in thermopile material are difficult to compensate.

Several micro flow sensors have used the time-of-flight measurement method [4-6]. Additionally, a few other methods using thermal waves have been devised [7-11]. A different low-drift approach is described in this paper, only requiring thin-film resistors. The presented thermal-wave balancing flow sensor applies sinusoidal currents on two heater resistors $[12,13]$, where a lock-in amplifier measures the resulting thermal waves on a single sensing resistor. The thermal-wave balancing measurement method and use of a lock-in amplifier rejects nearly all low frequency drift of the sensor, thus providing a flow sensor nearly independent of resistance drift and thermal gradient across the chip. However, a disadvantage is a slower response time of the sensor, since low frequencies have to be used in order to obtain sufficiently large thermalwave amplitudes. This can be mitigated by decreasing the distance between heater and sensor resistors, while reducing resistor size.

Figure 1 shows a schematic drawing of a thermal flow sensor and control system that exploits sinusoidal thermal waves, detected by a sensing resistor, to create a nearly offset-free low-drift power-feedback signal $\Delta P$ independent of 


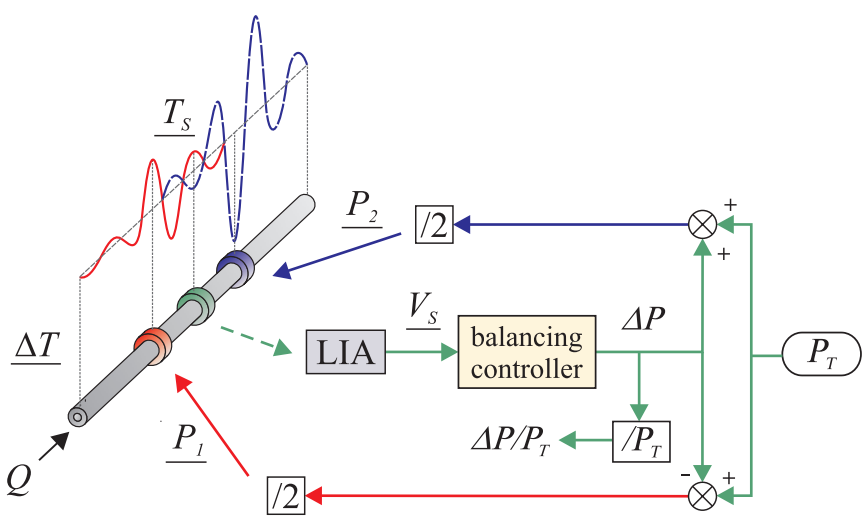

Figure 1. Control system for the thermal-wave balancing flow sensor.

$500 \mathrm{~nm} \mathrm{SiRN}$

$200 \mathrm{~nm} \mathrm{Al}$
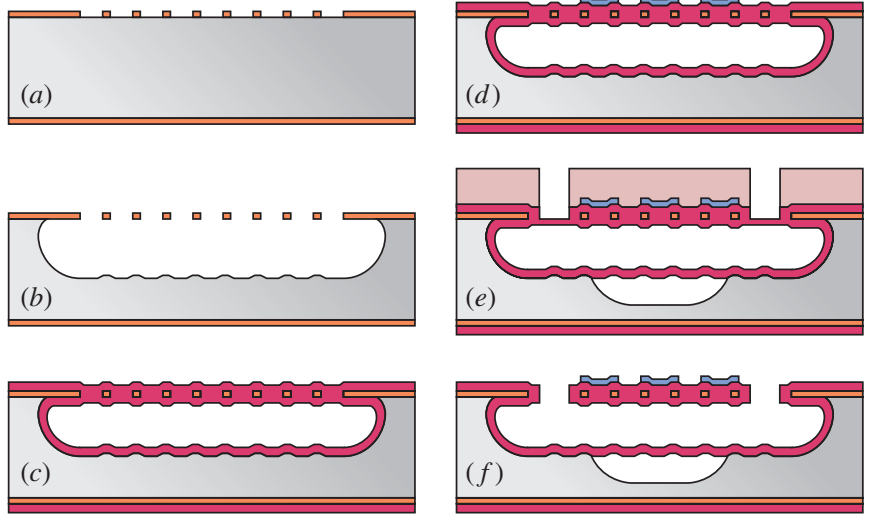

Figure 2. Process scheme for the fabrication of the thermal-wave balancing flow sensor.

sensor resistance value $R_{S}$. Two $180^{\circ}$ out-of-phase sinusoidal thermal waves and a superimposed static temperature field are generated by dissipating alternating currents $90^{\circ}$ outof-phase in two resistors, up- and downstream of a central sensing resistor. The heating powers $P_{1}$ and $P_{2}$ are controlled, with constant measurement of the resistance values of the heater resistors, $R_{1}$ and $R_{2}$, which can therefore drift without influencing sensor output. Thermo-electric offset voltages on interface wires are effectively eliminated by using alternating currents and a lock-in amplifier. The lock-in amplifier also eliminates offset signals due to slowly varying thermal gradients across the chip. The $180^{\circ}$ out-of-phase thermal waves cancel at $T_{S}$, after detection by the sensing resistor $R_{S}$, while minimizing $V_{S}$ by controlling $\Delta P$ between the two heater resistors, keeping the total dissipated heating power $P_{T}$ constant $[14,15]$. The amplitude of $V_{S}$ is controlled to a minimum by changing the power difference $\Delta P$. The exact amplitude of $V_{S}$ is therefore not important and the sensor resistance is allowed to drift. On applying a fluid flow the temperature distribution is changed by thermal advection. This results in the relation $\Delta P / P_{T}$ being dependent on the flow rate $Q$, being linear for small flow rates.

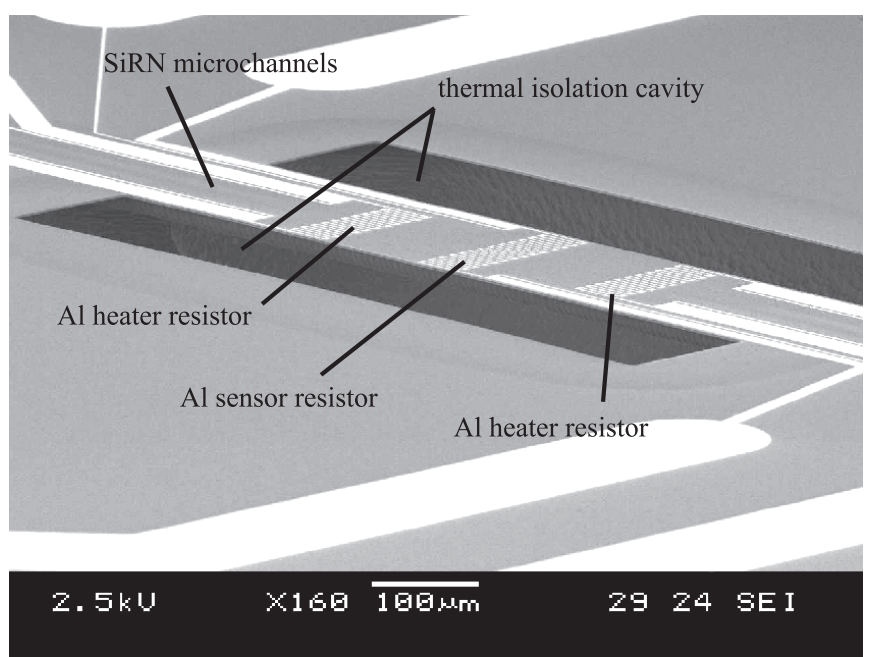

(a)

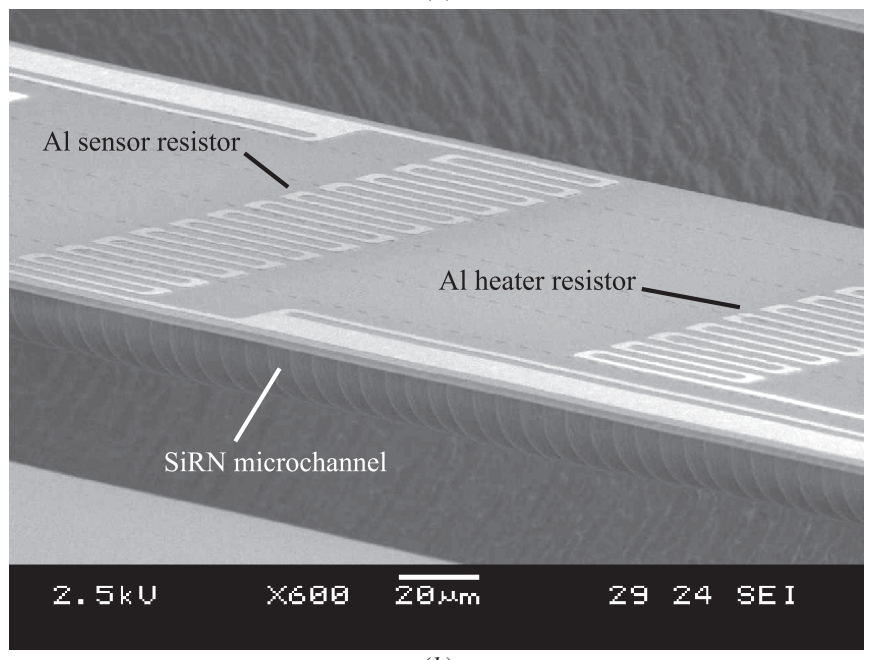

(b)

Figure 3. Micrographs of the thermal-wave balancing flow sensor, with $(a)$ sensor structure suspended over thermal isolation cavity and $(b)$ close-up of $\mathrm{Al}$ resistors.

\section{Sensor fabrication}

Thermal flow sensors were fabricated by using surface channel technology [16], for on-chip transport of fluid through mechanically strong freely suspended microchannels. The microchannels are fabricated below the surface of the wafer allowing for the integration of sensor elements in close proximity to the fluid.

Figure 2 shows the process scheme for the fabrication of the thermal-wave balancing flow sensor. Surface microchannels are created by isotropic dry etching, using high-density $\mathrm{SF}_{6}$ plasma with zero self-bias (figure 2(b)), through etch holes $2 \mu \mathrm{m}$ in width (figure $2(a)$ ), in a low-stress $500 \mathrm{~nm}$ silicon-rich silicon-nitride (SiRN) layer. The etch holes and inner surfaces of the microchannels are conformally coated by a second low-stress $1.2 \mu \mathrm{m}$ SiRN layer formed by using low-pressure chemical vapor deposition (LPCVD), resulting in completely sealed microchannels (figure $2(c)$ ), while leaving a planar substrate surface for the integration of $\mathrm{Al}$ resistors (figure $2(d)$ ). The surface microchannels are released by $\mathrm{SF}_{6}$ plasma etching, for thermal isolation from the 

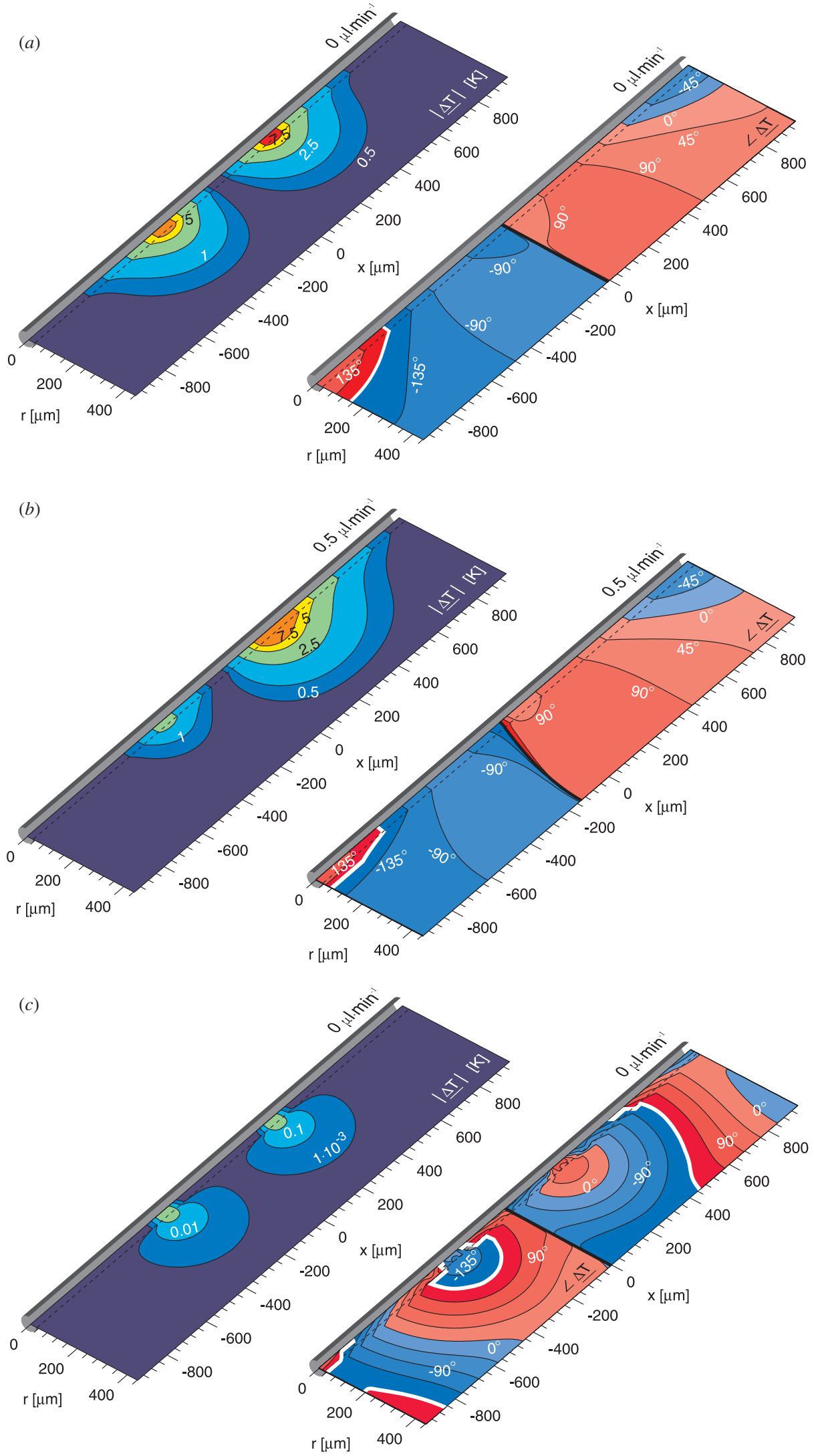

Figure 4. Calculated temperature amplitude (left) and phase (right) fields with $3 \mathrm{~mW}$ total power $P_{T}$ at $(a) 5 \mathrm{~Hz}$ without flow, (b) $5 \mathrm{~Hz}$ with $500 \mathrm{~nm} \mathrm{~min}^{-1}$ water flow and (c) $1 \mathrm{kHz}$ without flow. 
heat-conducting substrate (figure $2(f)$ ), with the photoresist mask protecting the sensor elements during the release (figure 2(e)).

Figure 3 shows scanning electron micrographs of the fabricated microchannel thermal flow sensor. The sensor contains five parallel $20 \mu \mathrm{m}$ diameter microchannels, spanning a $1.2 \mathrm{~mm}$ thermal isolation cavity. Up- and downstream $\mathrm{Al}$ heater resistors and an $\mathrm{Al}$ sensing resistor are integrated on top of the microchannels. The resistors have Kelvin contacts for accurate resistance measurements.

\section{Sensor modeling}

Thermal waves have been modeled for several microfluidic devices [17-20]. The thermal-wave balancing flow sensor in this paper has been modeled by using a two-dimensional cylindrical finite volume method (FVM) model constructed in MATLAB, solving the heat advection-diffusion equation for sinusoidal thermal waves, with fluid flow $v_{x}$ along the $x$-direction (1).

Material properties for both the fluid in the channel and surrounding air in the thermal isolation cavity are required, with $\rho$ the density, $c$ the heat capacity and $\kappa$ the thermal conductivity:

$$
\left(j 2 \omega \rho c+\rho c v_{x} \frac{\partial}{\partial x}-\nabla(\kappa \nabla)\right) T=P^{\prime \prime \prime} .
$$

Using complex sparse-matrix calculations, equation (1) can be solved on a cylindrical mesh of the sensor, resulting in a heat capacity matrix $\boldsymbol{B}$, a convection matrix $\boldsymbol{G}_{\boldsymbol{Q}}$ and a conduction matrix $\boldsymbol{G}_{\kappa}$, which can be solved effectively using the GaussSeidel method on equation (2):

$$
\begin{aligned}
& \left(j 2 \omega \cdot \boldsymbol{B}+\boldsymbol{Q} \cdot \boldsymbol{G}_{\boldsymbol{Q}}-\boldsymbol{G}_{\boldsymbol{\kappa}}\right) \underline{\vec{T}}_{e}=\underline{\vec{P}}_{e} \\
& \underline{\boldsymbol{Y}} \underline{\vec{T}}_{e}=\underline{\vec{P}}_{e}
\end{aligned}
$$

The column vectors $\underline{T}_{e}$ and $\underline{\vec{P}}_{e}$ contain, respectively, the resulting complex temperature and complex power input for each element in the FVM mesh. The $180^{\circ}$ out-ofphase sinusoidal thermal waves are given as two complex vectors at the heater positions, with amplitude determined by temperature-balancing.

Figure 4 shows calculated temperature fields, represented by the amplitude and phase of the complex field. Figure 4(a) shows the temperature field with no flow applied. Two thermal waves are generated at the location of the heater resistors, having equal amplitude at zero flow, where the phase plot shows that both thermal waves are actuated $180^{\circ}$ out of phase. In figure 4(b) $500 \mathrm{nl} \cdot \mathrm{min}^{-1}$ water flow is applied, where the downstream heater dissipates more than the upstream heater maintaining a minimum in the amplitude of the temperature at the sensing resistor. A slight asymmetry can be seen in the phase plot of figure $4(b)$, due to thermal advection in the microchannel.

The thermal waves at $5 \mathrm{~Hz}$ with no flow applied (figure 4(a)) extend only within one wavelength between the heater resistor and sensing resistor. Figure 4(c) shows the temperature field at $1 \mathrm{kHz}$, where more than one wavelength fits inside the sensor structure. The thermal penetration depth
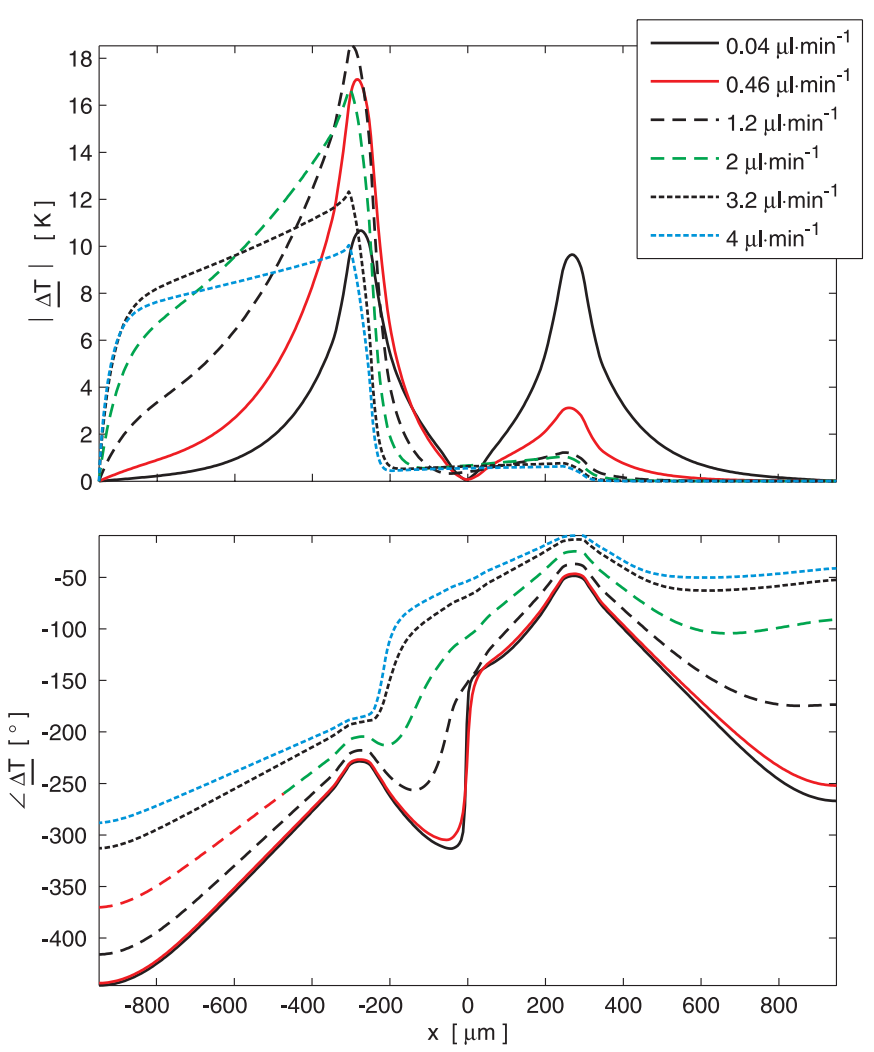

Figure 5. Calculated temperature amplitude and phase profiles along the microchannel at $5 \mathrm{~Hz}$ with $3 \mathrm{~mW}$ total heating power $P_{T}$ and various water flow-rates $Q$ in negative $x$-direction.

at this frequency decreases significantly, where most of the dissipated heat is retained by the heat capacity of the fluid inside the microchannel.

Figure 5 shows resulting temperature profiles along the microchannel from FVM model calculations. The temperature balancing of the thermal waves, minimizing the amplitude at the sensing resistor, was simulated for different flow rates $Q$. Figure 5 reveals that the amplitude in temperature at the sensing resistor can be made zero for flow rates up to $0.46 \mu \mathrm{l} \cdot \mathrm{min}^{-1}$. At flow rates of $1.2 \mu \mathrm{l} \cdot \mathrm{min}^{-1}$ and higher, a minimum can be obtained at the sensing resistor, but the temperature amplitude cannot be made completely zero, due to the large thermal advection from the upstream heater.

\section{Experimental results}

Fabricated thermal flow sensor chips were characterized using a chip holder for electrical and fluidic connections (figure 6). Water flow was applied by adjusting an elevation head, while the actual flow rate was calibrated by microbalance weighing. The measurements were controlled by MATLAB. The heater resistors were heated by applying an alternating current (HP $3245 \mathrm{~A}$ ). The heating power was controlled accurately by reading the voltage drop over the heater resistors using multimeters (HP 34401A) and by adjusting the thermal-wave amplitude accordingly. A small direct current was passed through the sensing resistor (Keithley 237), while the $2 \omega$ component in the voltage drop over the sensing resistor was measured by using a DSP lock-in amplifier (SRS 830). 


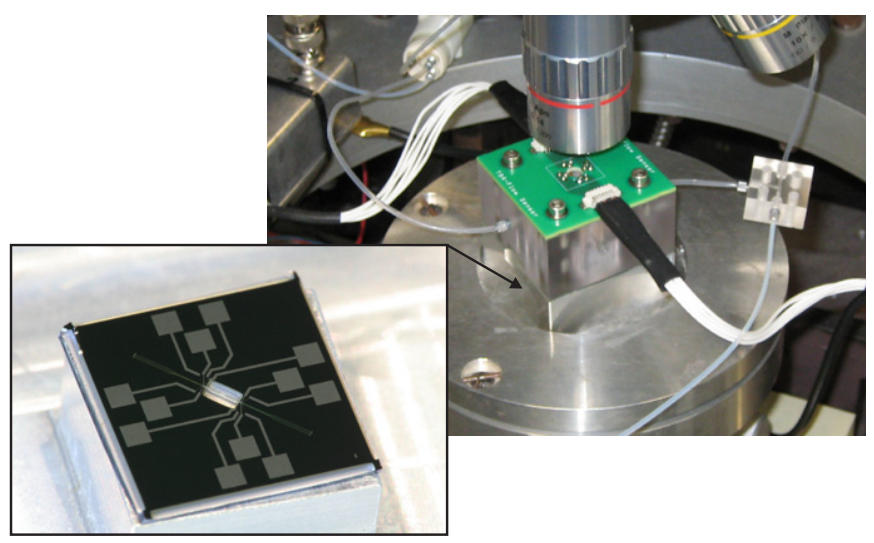

Figure 6. Electrical and fluidic interconnection to the sensor chip. Inset shows a photograph of the thermal-wave balancing flow sensor chip.
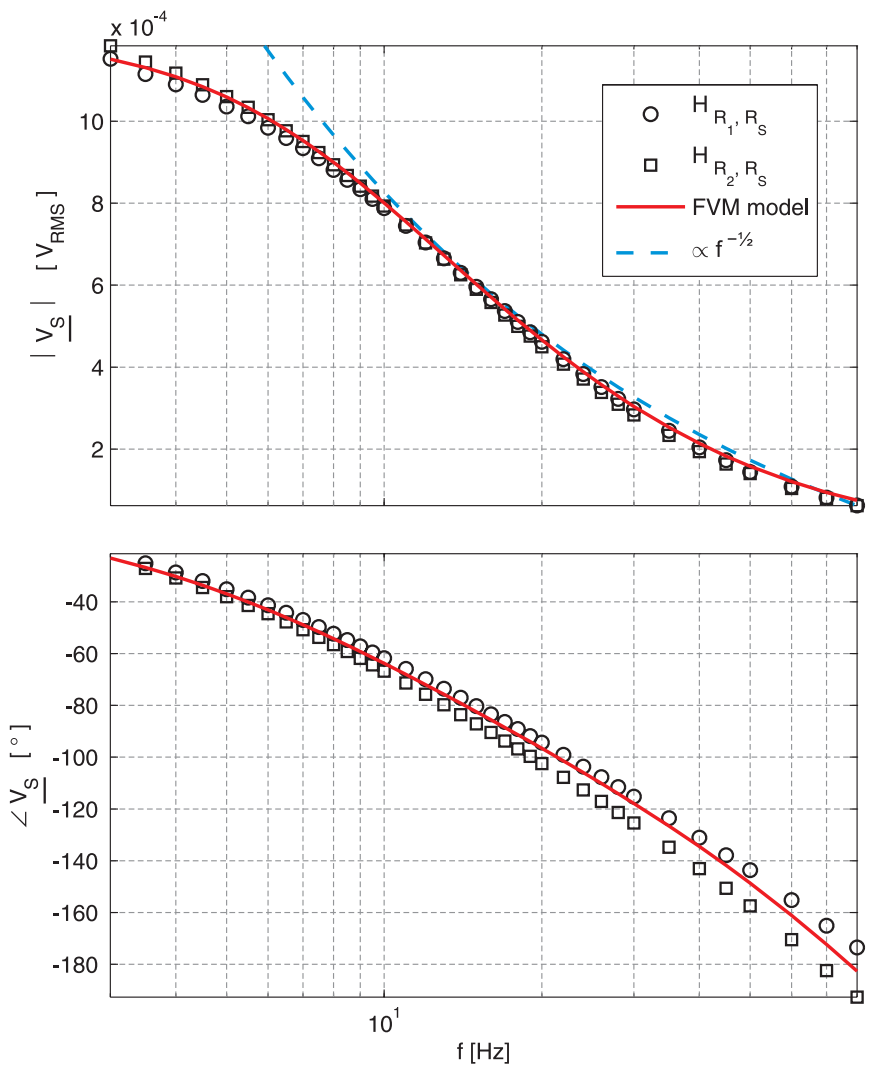

Figure 7. Frequency response of each heater resistor $R_{1}, R_{2}$ to the sensing resistor $R_{S}$ at $0.4 \mathrm{~mW}$ power $P_{T}$ compared to FVM model calculations and thermal-penetration depth dependency.

Figure 7 shows the open-loop frequency response simulated and measured for each heater resistor to the sensing resistor without flow. The amplitude is proportional to $f^{-\frac{1}{2}}$ for frequencies higher than $10 \mathrm{~Hz}$, determined by the thermal penetration depth of the sinusoidal heat wave, where the thermal penetration depth approximately equals the distance between resistors at $10 \mathrm{~Hz}$. A small phase difference between both heaters can be observed in figure 7 for higher frequencies, indicating a small asymmetry in the flow sensor.

Figure 8 shows the sensor response on the power difference $\Delta P$ between both heaters. Minimum amplitude for
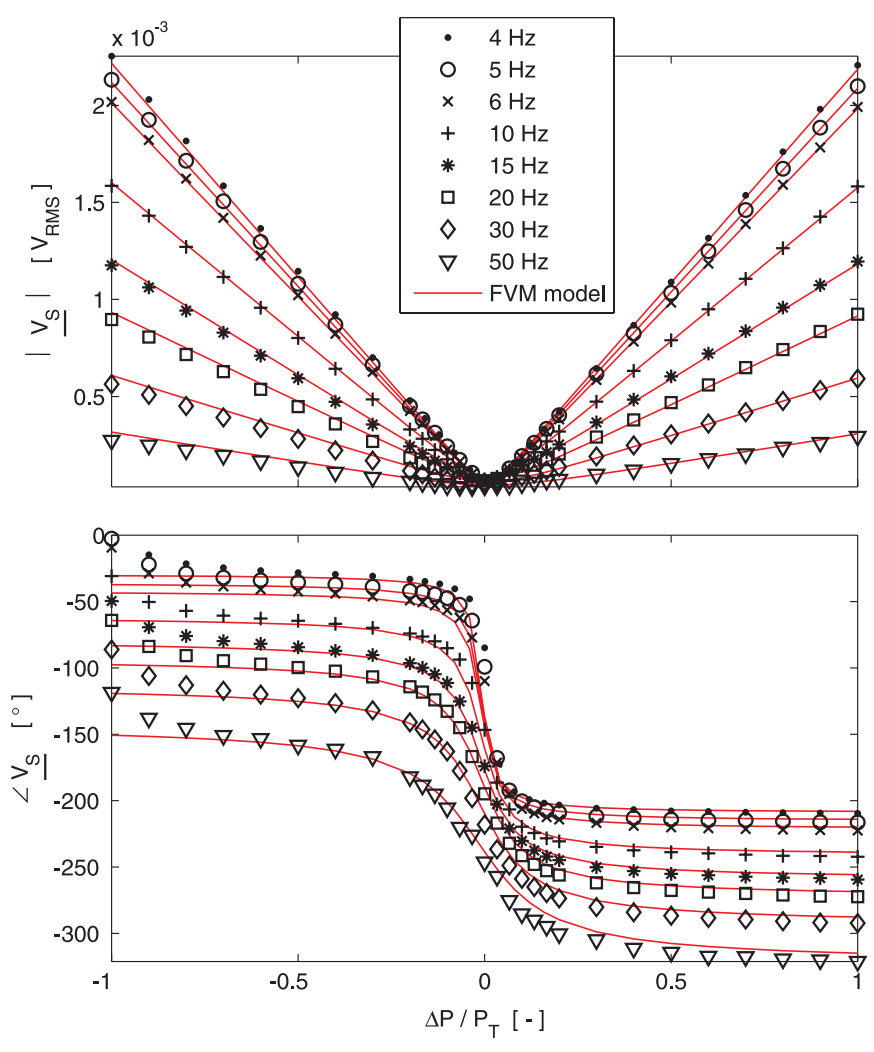

Figure 8. Open-loop sensing resistor response on power difference $\Delta P$ without flow at various frequencies with $0.8 \mathrm{~mW}$ total applied heating power $P_{T}$ compared to FVM model calculations.

$V_{S}$, independent of frequency, is obtained when both heaters dissipate equal amount of power. The determination of the minimum in amplitude is independent of the sensor resistance value $R_{S}$ and is theoretically offset and drift-free. However, asymmetries in the sensor structure can still give rise to an offset in $\Delta P$, which seems to be more apparent in closeloop operation. The decrease in slope of the phase of $V_{S}$ near zero $\Delta P / P_{T}$ at higher frequencies (figure 8 ) is caused by the measured sensor asymmetry. The FVM model results only show this decrease in slope when the phase difference from figure 7 is taken into consideration.

The position of the minimum in the amplitude of $V_{S}$ changes with applied flow rate. Figure 9 shows measured and simulated open-loop response on $\Delta P$ to various water flow rates. These open-loop measurements show that it is difficult to use the phase of $V_{S}$ as a control signal. In figure 8 the phase decreases with increasing frequency. The FVM model indicates that this is also dependent on the type of fluid in the microchannel. This is because thermal conduction and heat capacity determine the phase of the temperature at the heater resistors, which does not need to be exactly the same as the phase in the power dissipated in the heaters.

Figure 9 shows the sensor response of the flow sensor obtained by determining the $\Delta P$ required for minimum amplitude of $V_{S}$ for various flow rates. The sensor output $\Delta P / P_{T}$ appears to be independent of operation frequency as well as total heating power. A nearly linear sensor output is obtained up to about $500 \mathrm{nl} \cdot \mathrm{min}^{-1}$. The FVM model accurately indicates that at higher flow rates temperature 


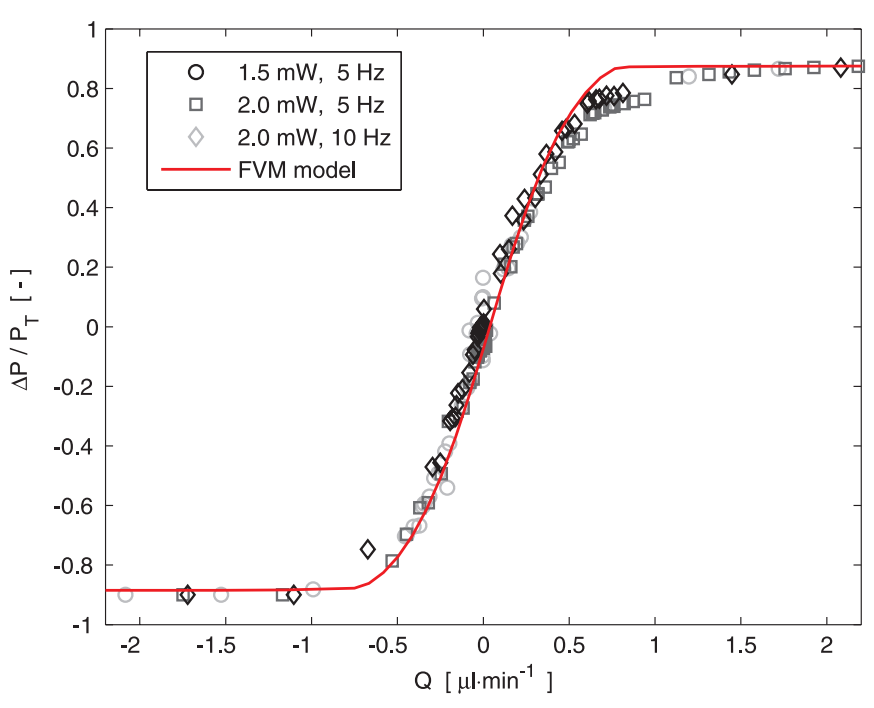

Figure 9. Sensor output $\Delta P / P_{T}$ response on water flow rate $Q$ at different frequencies and total heating power, compared to FVM model results.

gradients near the silicon substrate force the heater temperature to decrease (figure 5).

A balancing controller, as depicted in figure 1, was implemented in MATLAB, applying gradient descent to obtain $\Delta P$ at minimum amplitude of $V_{S}$. The gradient is determined by two measurement points $\left(\Delta P_{n} \pm \delta P\right)$ close to $\Delta P_{n}$. Using small step-size for convergence, $\Delta P_{n+1}$ is placed in the opposite direction to the gradient. Convergence can be slow when $\Delta P_{n}$ is far from minimum amplitude. A direct linear fit to find the minimum, when large changes in flow rate occur, can be made if during sensor operation an estimate of the absolute value of the minimum amplitude of $V_{S}$ can be made. Accurate convergence has to be obtained by gradient descent, if the direct fit is not entirely accurate. The question remains of how to optimally decide at what change in flow rate the linear fit should be applied. Figure 10 shows that direct linear fit combined with gradient descent has a much faster response than when only using gradient descent. A sample time $n$ of only $50 \mathrm{~s}$ could be obtained. This is mostly limited by the DSP lock-in amplifier (SRS 830). Much faster sampling could be obtained by using dedicated electronics. Ultimately, the sample time is limited by the frequency of the thermal waves measured with the lock-in amplifier, where an optimum has to be found between required sample time and the decrease in amplitude measured by the sensing resistor, due to a smaller thermal penetration depth.

A drift measurement for $498 \mathrm{~h}$ was conducted without water in the microchannel in an ambient environment. Figure 11 shows the power spectral density of the heater resistors and the drift in the sensor output $\Delta P$. The heater resistors clearly show $1 / f^{-\alpha}$ noise spectra, while the power spectral density for $\Delta P$ is nearly flat. Measurement data of $\Delta P$ indicate that thermal gradients over the chip are not measured. For instance, no temperature fluctuation due to day and night rhythm was observed in the time signal of $\Delta P$, while this could be observed in a slight change in resistance values of the heater resistors.
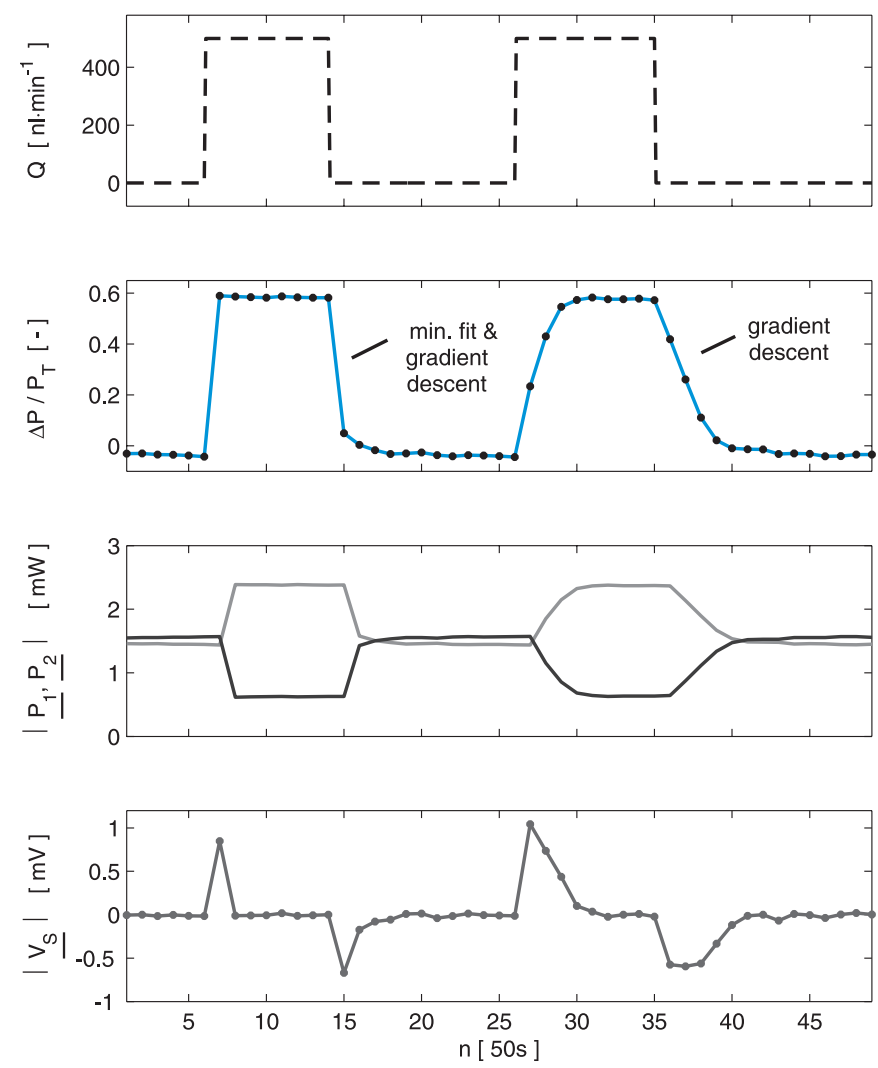

Figure 10. Sensor control-system response on changing flow rate $Q$ at $3 \mathrm{~mW}$ total heating power $P_{T}$. During the second change in flow only the gradient descent method is used, resulting in a significant slower response. 


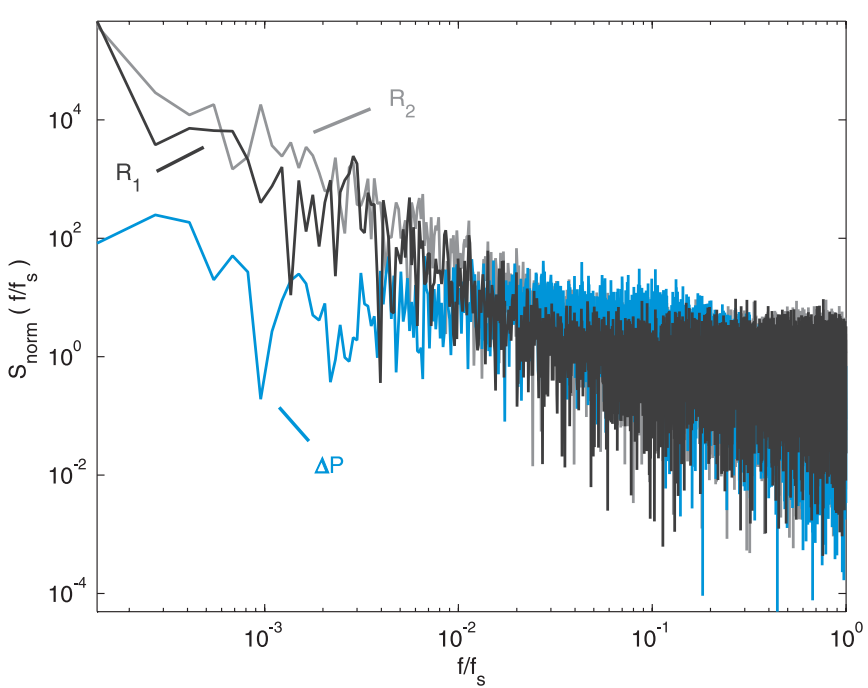

Figure 11. Drift measurement for $498 \mathrm{~h}$ with $8.210^{-3} \mathrm{~Hz}$ sampling frequency $f_{s}$, showing normalized power spectral density $S_{\text {norm }}$ at $2 \mathrm{~mW}$ total power $P_{T}$

\section{Conclusions}

A microchannel thermal flow sensor with integrated $\mathrm{Al}$ resistors has been fabricated successfully. The flow sensor is capable of measuring water flow rates with $\mathrm{nl} \cdot \mathrm{min}^{-1}$ precision up to about $500 \mathrm{nl} \cdot \mathrm{min}^{-1}$ full scale, applying the temperaturebalancing concept [14] using thermal waves. A sensor output independent of operation frequency, resistance values and total heating power has been obtained. The thermal operation of the flow sensor is well understood by the dynamic FVM model of the flow sensor, giving near exact fits to measurement results. A power-feedback control system based on gradient descent shows stable operation independent of operation frequency and thermal properties.

A disadvantage of the measurement method is the slow response time, because relatively low frequencies have to be used in combination with long averaging times. Effects of thermal gradient across the chip, resistance drift and thermo-electric offset voltages are however eliminated by using a relatively simple sensor structure. The decrease of the thermal penetration depth with frequency indicates that faster operation frequencies can be achieved in much smaller devices, measuring e.g. small flows in nanochannels [21].

\section{Acknowledgment}

The authors would like to thank the Dutch Technology Foundation (STW) for financial support through the low-drift micro-flowsensors project (TET.6634).

\section{References}

[1] Wu S, Lin Q, Yuen Y and Tai Y-C 2001 MEMS flow sensors for nano-fluidic applications Sensors Actuators A 89 152-8
[2] Dijkstra M, de Boer M J, Berenschot J W, Lammerink T S J, Wiegerink R J and Elwenspoek M 2008 Miniaturized thermal flow sensor with planar integrated sensor structures on semicircular surface channels Sensors Actuators A $1431-6$

[3] Dijkstra M, Lammerink T S J, de Boer M J, Berenschot J W, Wiegerink R J and Elwenspoek M 2008 Low-drift flow sensor with zero-offset thermopile-based power feedback Proc. DTIP pp 247-50

[4] Berthet H, Jundt J, Durivault J, Mercier B and Angelescu D 2011 Time-of-flight thermal flowrate sensor for lab-on-chip applications Lab. Chip 11 215-23

[5] Ćerimović S, Talić A, Kohl F, Beigelbeck R, Schalko J and Jachimowicz A 2009 Micromachined flow sensor enabling electrocalorimetric and TOF transduction Procedia Chem. 1 132-5

[6] Meng E, Li P-Y and Tai Y-C 2008 A biocompatible parylene thermal flow sensing array Sensors Actuators A 144 18-28

[7] Ćerimović S, Schalko J, Jachimowicz A, Talić A, Beigelbeck R and Kohl F 2008 Novel flow sensors based on a two-state controller scheme Proc. IEEE Sensors pp 1163-6

[8] Lambert D K and Harrington C R 1985 An air flow sensor based on interface thermal wave propagation J. Appl. Phys. 59 59-65

[9] Rachalski A 2013 Absolute measurement of low gas flow by means of the spectral analysis of the thermal wave Rev. Sci. Instrum. 84025105

[10] Bruschi P, Navarrini D and Piotto M 2004 A flow sensor for liquids based on a single temperature sensor operated in pulse mode Sensors Actuators A 110 269-75

[11] Al-Salaymeh A and Durst F 2004 Development and testing of a novel single-wire sensor for wide range flow velocity measurements Meas. Sci. Technol. 15 777-88

[12] Lammerink T S J, Tas N R, van Honschoten J W, Krijnen G J M, van Baar J J and Elwenspoek M 2001 AC-driven temperature-balance flow sensor Proc. 11th Int. Conf. on Solid State Sensors and Actuators (Munich, Germany; June 2001)

[13] Dijkstra M, Lammerink T S J, Pjetri O, de Boer M J, Berenschot J W, Wiegerink R J and Elwenspoek M 2008 AC-driven temperature-balancing flow sensor with zero-offset power feedback Proc. MME pp 401-4

[14] Lammerink T S J, Tas N R, Krijnen G J M and Elwenpoek M 2000 A new class of thermal flow sensors using $\Delta T=0$ as a control signal 19th MicroMechanics Europe Workshop (Aachen, Germany; September 2008) pp 525-30

[15] Bruschi P, Diligente A, Navarrini D and Piotto M 2005 A double heater integrated gas flow sensor with thermal feedback Sensors Actuators A 123-4

[16] Dijkstra M, de Boer M J, Berenschot J W, Lammerink T S J, Wiegerink R J and Elwenspoek M 2007 A versatile surface channel concept for microfluidic applications J. Micromech. Microeng. 17 1971-7

[17] Reyes-Romero D F, Cubukcu A S and Urban G A 2012 Simulation of the oscillatory excitation of a thermal flow sensor 1st Int. Conf. on MicroFluidic Handling Systems (Enschede, The Netherlands; October 2012) pp 22-25

[18] Lambert D K 1993 Mass flow sensing with heat waves: the effect of gas pressure Int. J. Heat Mass Transfer 36 2623-34

[19] Jonsson U G and Andersson O 1998 Investigation of the low- and high-frequency response of $3 \omega$-sensors used in dynamic heat capacity measurements Meas. Sci. Technol. 9 1873-85

[20] Handford P M and Bradshaw P 1989 The pulsed-wire anemometer Exp. Fluids 7 125-32

[21] Mathwig K and Lemay S G 2013 Pushing the limits of electrical detection of ultralow flows in nanofluidic channels Micromachines 4 138-48 